\title{
Pelvic recurrence after Miles' operation for anastomotic recurrence in a patient with stage I rectal cancer invading the proper muscle layer: Case report
}

\author{
TATSUHIKO HOSHIKAWA ${ }^{1}$, MASAYA MUKAI ${ }^{1}$, YASUHISA OIDA $^{1}$, TAKAYUKI TAJIMA ${ }^{1}$, \\ GORYU MORIKAWA ${ }^{1}$, TOMOKI NAKAMURA ${ }^{1}$, MEGUMI MOTOJYUKU ${ }^{1}$, \\ MASATO NAKAMURA ${ }^{2}$ and HIROYASU MAKUUCHI ${ }^{3}$

\begin{abstract}
Departments of ${ }^{1}$ Surgery and ${ }^{2}$ Pathology, Tokai University Hachioji Hospital, Ishikawa-cho, Hachioji, Tokyo 192-0032;
${ }^{3}$ Department of Surgery, Tokai University School of Medicine, Bohseidai, Isehara, Kanagawa 259-1193, Japan
\end{abstract}

Received November 1, 2006; Accepted November 24, 2006

\begin{abstract}
We performed D2 low anterior resection in a patient with stage I rectal cancer [pathological diagnosis: proper muscle (pm) invasion, n0, lymphatic invasion (ly), (-); venous invasion (v), (-); anal margin, (-)]. The tumor recurred at the anastomotic site approximately one year later and was treated with Miles' operation [pm, n0, ly (+); v (-); deep border of the primary tumor (-)]. The tumor marker CEA increased to $50.4 \mathrm{ng} / \mathrm{ml}$ at four months after surgery and pelvic local recurrence was detected. Since then, the patient has been receiving chemoradiotherapy on an out-patient basis. Cytokeratin immunostaining of all the lymph nodes collected during the two operations showed clusters of occult neoplastic cells (ONCs) in the perinodal fat around the nodes harvested at the first operation. These findings suggest that the risk of local recurrence of rectal cancer is increased even in stage I disease if ONCs are found in the perinodal fat. Further studies are required to examine the relationship between local recurrence and extranodal ONCs in patients with primary rectal cancer.
\end{abstract}

\section{Introduction}

Low rectal cancer is known to recur in the perineum after rectal amputation and anastomosis or at presacral sites in the

Correspondence to: Dr Tatsuhiko Hoshikawa, Department of Surgery, Tokai University Hachioji Hospital, Ishikawa-cho 1838, Hachioji, Tokyo 192-0032, Japan

E-mail: hoshikawa.tatsuhiko@hachioji-hosp.tokai.ac.jp

Abbreviations: LNs, lymph nodes; ONCs, occult neoplastic cells; pm, proper muscle; am, anal margin; om, oral margin

Key words: colorectal cancer, pelvic local recurrence, proper muscle invasion, occult neoplastic cells, extranodal tumor deposit, stage I cancer pelvis after low anterior resection (1-3). The 5-year survival rate of patients with stage I rectal cancer is reported to be 90-95\% and the incidence of postoperative distant metastasis/ recurrence is only $5-10 \%$. The incidence of postoperative local recurrence in the pelvic cavity is also quite low, being only a few percent (4-6). Possible risk factors for the postoperative local recurrence of low rectal cancer including stage I disease are: i) an inadequate resection margin of $<2 \mathrm{~cm}$, ii) seeding of tumor cells by large bowel perforation due to operative manipulation or inadequate suturing, iii) implantation of viable tumor cells in the alimentary tract by mechanical anastomosis, and iv) occult microscopic tumor invasion, including visually undetectable microscopic lymph node metastasis and microscopic deposits in the pelvic fat (7-9). Many studies have also shown a close relationship between the detection of cytokeratin-positive occult neoplastic cells (ONCs) in lymph node sinuses (intranodal ONCs) far from the primary tumor and distant metastasis/recurrence of n0 disease (10-16).

We performed low anterior resection in a patient with stage I rectal cancer, but recurrence was detected at the anastomosis one year after surgery, so Miles' operation was performed. Unfortunately, a tumor marker was found to be increased and intrapelvic local recurrence was detected four months after the second operation. Since then, the patient has received chemoradiotherapy on an out-patient basis. We reviewed the clinical course of this patient and the published reports about local recurrence of stage I rectal cancer with proper muscle (pm) invasion.

\section{Patient and clinical course}

Diagnosis of stage I rectal cancer. A 71-year-old man presented with fresh melena in April 2005. He had no notable medical or surgical history.

Laboratory findings. No anemia or jaundice was noted. None of the tumor markers that were examined showed an increase. No other abnormal findings were observed.

Physical examination. The abdomen was soft and flat, with no abnormalities. Digital rectal examination showed a small, 


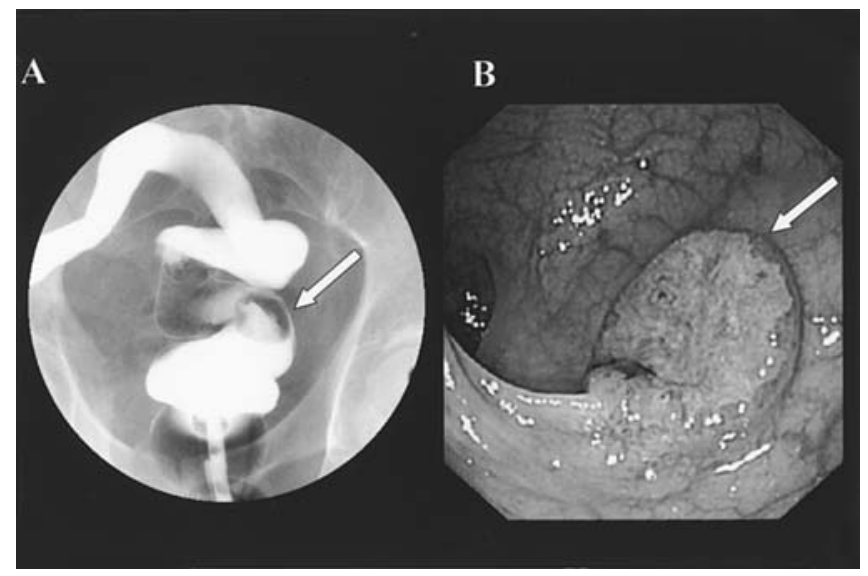

Figure 1. Barium enema shows a small type II cancer on the left anterior wall of the rectum ( $\mathrm{Ra}$ ) about $7 \mathrm{~cm}$ from the anal verge, which extends over approximately one-third of the rectal circumference (A, arrow). The biopsy specimen obtained during colonoscopy allowed a diagnosis of adenocarcinoma to be made (B, arrow).

hard nodule that extended over approximately one-third of the circumference of the rectum and was located approximately $7 \mathrm{~cm}$ from the anal verge.

Gastroscopy findings. No abnormalities were noted.

Barium enema findings. A small type II cancer was observed on the left anterior wall of the lower rectum (Ra). It was located approximately $7 \mathrm{~cm}$ from the anal verge and covered approximately one-third of the rectal circumference. Since the adventitia was intact, the depth of invasion was considered to be pm (Fig. 1A).

Total colonoscopy findings. A small type II cancer was observed in the lower rectum ( $\mathrm{Ra})$. It was located approximately $7 \mathrm{~cm}$ from the anal verge and extended over approximately one-third of the rectal circumference. Biopsy gave a diagnosis of adenocarcinoma. No other abnormalities were noted (Fig. 1B).

Ultrasound and CT findings. There were no findings suggestive of lymph node metastasis, such as enlarged paraaortic lymph nodes (LNs), no involvement of organs such as the liver and lungs, and no invasion of other structures around the primary tumor.

Based on these findings, the patient was diagnosed as having stage I rectal cancer (pm and $\mathrm{n} 0$ ). He underwent D2 low anterior resection on April 15, 2005.

Pathological findings. Pathological examination showed that the tumor was a well-differentiated adenocarcinoma in stage I with the following characteristics: depth of tumor invasion; pm, n0, lymphatic invasion (ly), (-); venous invasion (v), (-); anal margin (am), (-); and oral margin (om), (-) (Fig. 2A).

Anastomotic recurrence after low anterior resection. Approximately one year after the operation, digital rectal examination revealed a palpable induration approximately $3 \mathrm{~cm}$ in diameter at the anastomosis.

Total colonoscopy findings. A small tumor on the left anterior wall of the residual rectum at the anastomosis, which was type II and spread over approximately one-third of the rectal circumference. Biopsy gave a diagnosis of recurrent

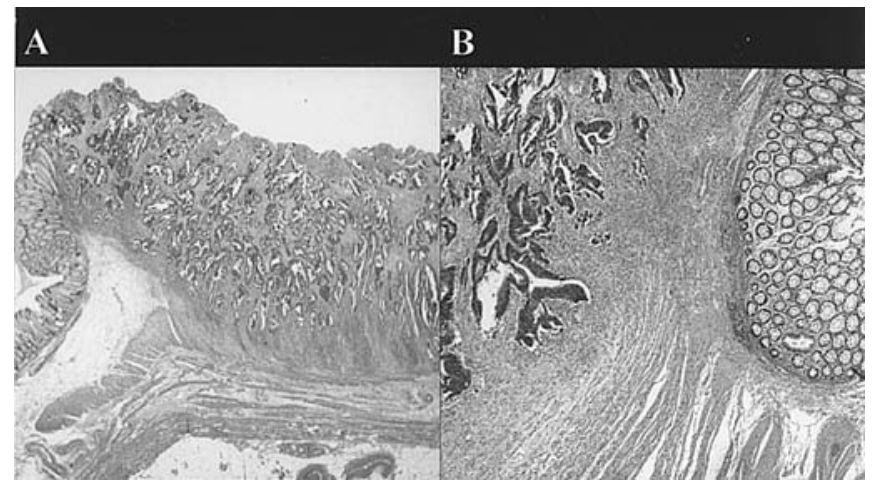

Figure 2. (A) Pathological examination showed that the lesion was a welldifferentiated adenocarcinoma. The tumor was stage I with invasion of the proper muscle layer (pm) [n0, lymphatic invasion (ly), (-); venous invasion (v), (+); and anal margin (am) and oral margin (om) of the resected specimen, (-)]. (B) Pathological examination showed that the lesion was a welldifferentiated adenocarcinoma with the following features: pm, n0, ly (+), v $(-)$, am and om of the resected specimen (-), and deep border of the primary tumor (-).

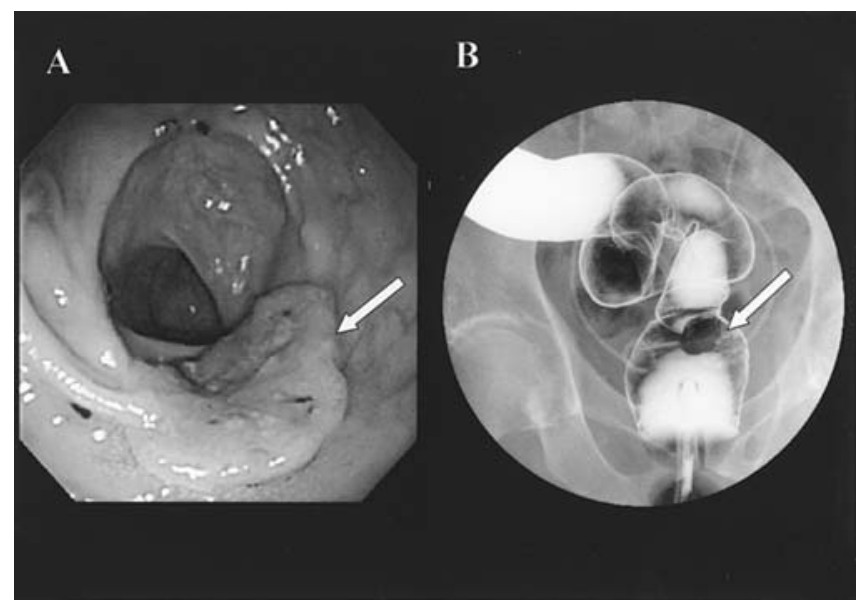

Figure 3. Total colonoscopy showed a small lesion on the left anterior wall of the rectum distal to the anastomosis, which extended over approximately one-third of the rectal circumference. Biopsy gave a diagnosis of recurrent adenocarcinoma (A, arrow). Barium enema showed a small anastomotic recurrence on the left anterior wall of the residual rectum $(\mathrm{Rb})$ approximately $3 \mathrm{~cm}$ from the anal verge, which covered approximately one-third of the rectal circumference (B, arrow).

adenocarcinoma. No abnormalities were observed on the oral side of the anastomosis (Fig. 3A).

Barium enema findings. A small type II cancer was observed on the left anterior wall of the residual lower rectum $(\mathrm{Rb})$ at approximately $3 \mathrm{~cm}$ from the anal verge. It extended over approximately one-third of the rectal circumference. Since no infiltration of the adventitia was observed, the lesion was diagnosed as an anastomotic recurrence with $\mathrm{pm}$ invasion (Fig. 3B). There were no other abnormal findings, including no increase of tumor marker levels and no evidence of distant metastasis and pelvic recurrence. Miles' operation was performed on March 2, 2006.

Pathological findings. Pathological examination showed that the tumor was a well-differentiated adenocarcinoma with the following characteristics: depth of tumor invasion; pm, 


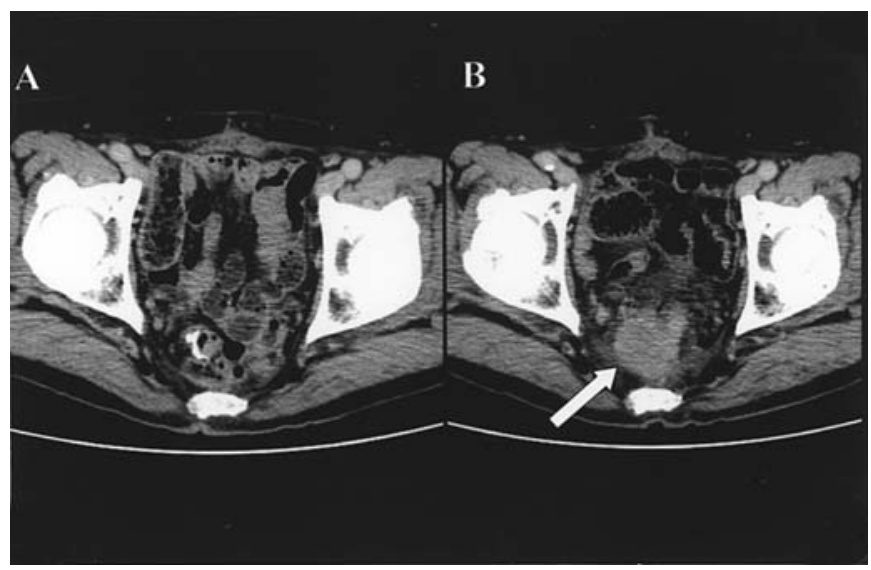

Figure 4. The patient was discharged after the absence of postoperative complications was confirmed by CT scanning (A). Follow-up CT showed an irregular low-density area approximately $6 \mathrm{~cm}$ in diameter in the presacral space. Based on this finding and marked elevation of CEA, he was diagnosed as having pelvic local recurrence (B, arrow).

n0, ly (+), v (-), am (-), om (-), and deep border of the primary tumor (-) (Fig. 2B).

Pelvic recurrence after Miles' operation. The patient was discharged in good condition after the absence of postoperative complications was confirmed by $\mathrm{CT}$ scanning and other examinations (Fig. 4A). However, follow-up CT performed three months after discharge showed an irregular low-density area in the presacral space and the CEA level was markedly increased to $50.4 \mathrm{mg} / \mathrm{dl}$. He was therefore diagnosed as having pelvic local recurrence (Fig. 4B). Radiation therapy for the pelvic tumor was started in August 2006, and was followed by systemic chemotherapy.

Additional pathological findings and immunohistochemistry. Although the primary rectal cancer and the anastomotic recurrence were pathologically re-examined in detail, no additional findings were noted regarding the depth of tumor invasion or vascular invasion. Cytokeratin immunohistochemical staining was performed on all the LNs harvested during the two operations. This showed that the LNs and lymph node sinuses did not contain any ONCs positive for cytokeratin (intranodal ONCs), but that the perinodal fat around LNs removed at the first laparotomy contained numerous clusters of ONCs (extranodal ONCs) (10-16) (Fig. 5).

\section{Discussion}

The 5-year survival rate of stage I rectal cancer is reported to be $90-95 \%$ (4-6). Postoperative distant metastasis/recurrence of such tumors is relatively uncommon and the rate of local recurrence in the pelvic cavity is also very low (1-3). Our patient who underwent typical D2 resection had none of the recognized risk factors for systemic dissemination resulting in metastasis/recurrence, since his primary tumor showed minimal invasion (pm), lymph node metastasis was negative on pathological examination after routine $\mathrm{H} \& \mathrm{E}$ staining (n0), and there was no lymphatic or vascular invasion (ly0 and v0).
A

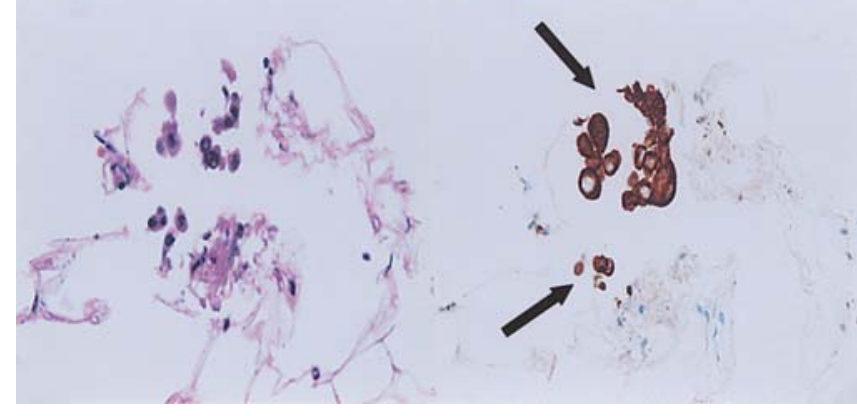

Figure 5. Many clusters of occult neoplastic cells (ONCs) positive for cytokeratin were observed in the perinodal fat around lymph nodes harvested during the first operation (extranodal ONCs) [(A) H\&E stain x200; (B), AE1/3 stain x200, arrows].

Therefore, the possibility that surgical manipulation of the tumor caused the implantation of cancer cells in the large bowel distal to the anastomosis cannot be excluded (7-9). This is based on the assumption that small clusters of cells separated from the primary tumor by surgical manipulation may be implanted at sites of mucosal damage distal to the primary lesion. Prophylactic use of perioperative rectal lavage to avoid such recurrence has been described in many reports, and some authors have referred to the need to achieve a cytocidal antitumor effect (7-9). However, even if clusters of cancer cells are implanted in the residual large bowel during mechanical anastomosis, they should have been completely removed by Miles' operation as long as the tumor was actually pm invasion. It has also been suggested that microscopic lymph node metastasis around the distal stump of the rectum may lead to infiltration of the anastomosis (7-9). In the present case, however, postoperative pelvic CT scans showed no lesions near the anastomosis after the first procedure. Only colonoscopy showed a flat lesion that was $<30 \mathrm{~mm}$ in diameter as evidence of recurrence on the left anterior wall distal to the anastomosis. It seems quite unlikely that residual tumor cells from a lymph node would suddenly infiltrate the rectal mucosa to produce such a tumor. Since many clusters of ONCs were noted in the perinodal fat around the LNs harvested during the first operation, it seems more likely that recurrence in our patient was due to inappropriate surgical manipulation of the distal rectum and mesorectum or occurred because the distance from the primary tumor to the distal resection margin was inadequate. However, a distance of at least $2 \mathrm{~cm}$ was maintained during distal resection with am (-), which conforms to the Japanese Rules for Handling Colon and Rectal Cancer $(5,6)$. Therefore, it seems that low rectal anastomosis has to be performed with great care, even when the cancer is only stage I.

Various studies have shown that the detection of intranodal ONCs in LNs indicates the systemic spread of cancer cells more accurately than the ly and $\mathrm{v}$ status of the primary tumor (10-16). In fact, many clusters of extranodal ONCs were observed in the dissected perinodal fat of the present patient, despite the tumor being ly0 and v0. A close relationship between tumor cells in the extranodal fat and local recurrence has long been reported for breast cancer, esophageal cancer 
and lung cancer (17-19). Small deposits of such tumors, including rectal cancer, may develop into larger lesions that can be easily detected by routine H\&E staining when perineural invasion or tumor spread into the resected fat is examined at the distal stump $(8,20-24)$. Detection of ONCs that can only be confirmed by immunohistochemistry has been reported to indicate a high risk of the recurrence of stage I/II n0 disease after curative resection (10). Among ONCs, it is thought that single cells or clusters ( $\leq 10$ ONCs) can survive more easily and become established in a new microenvironment to form micrometastatic foci that lead to local recurrence in the pelvis (10). Further studies are necessary to determine whether it is necessary to perform additional wide resection during Miles' operation for stage I/II n0 disease, or perform radiation therapy and/or systemic consolidation chemotherapy in patients who have clusters of extranodal ONCs in the perinodal fat as in the present case.

\section{Acknowledgements}

This study was supported by grants from the Occult Neoplastic Cells Research and Study Group (No. 2006-5048; Tokai University Hachioji Hospital, Hachioji, Tokyo, Japan) and the Research and Study Program of Tokai University Educational System General Research Organization (No. 2005-41; Tokai University Hospital, Isehara, Kanagawa, Japan).

\section{References}

1. Hurst PA, Prout WG, Kelly JM, Bannister JJ and Walker RT: Local recurrence after low anterior resection using the staple gun. Br J Surg 69: 275-276, 1982.

2. Tomoda $\mathrm{H}$ and Furusawa M: Local recurrence after curative anterior resection for carcinoma of the rectum. Jpn J Surg 16: 377-379, 1986.

3. Suzuki K, Muto T and Sawada T: Prevention of local recurrence by extended lymphadenectomy for rectal cancer. Jpn J Surg 25: 795-801, 1995.

4. Journal of Health and Welfare Statistics, Health and Welfare Statistics Association, Tokyo, 52: 45-47, 2005.

5. Multi-Institutional Registry of Large Bowel Cancer in Japan. Cases treated in 1994. Japanese Society for Cancer of the Colon and Rectum, Tokyo, 23, 2002.

6. Guideline of Large Bowel Cancer in Japan. Japanese Society for Cancer of the Colon and Rectum, Tokyo, 2005.

7. Syfan J, Averbuch F, Koltun L and Benyamin N: Effect of rectal stump washout on the presence of free malignant cells in the rectum during anterior resection for rectal cancer. Dis Colon Rectum 43: 1710-1712, 2000.

8. Prabhudesai A, Arif S, Finlayson CJ and Kumar D: Impact of microscopic extranodal tumor deposits on the outcome of patients with rectal cancer. Dis Colon Rectum 46: 1531-1537, 2003.

9. Terzi C, Ünek T, Sagol O, Yilmaz T, Füzün M, Sökmen S, Ergör G and Küpelioglu A: Is rectal washout necessary in anterior resection for rectal cancer? A prospective clinical study. World J Surg 30: 233-241, 2006.
10. Mukai M: Occult neoplastic cells and malignant microaggregates in lymph node sinuses: review and hypothesis. Oncol Rep 14: 173-175, 2005

11. Mukai M, Sato S, Ninomiya H, Wakui K, Tsuchiya K, Komatsu N, Nakasaki H and Makuuchi H: Recurrence and 5-FU sensitivity of stage III/Dukes' C colorectal cancer with occult neoplastic cells in lymph node sinuses. Oncol Rep 14: 1165-1169, 2005.

12. Mukai M, Sato S, Ninomiya H, Wakui K, Tsuchiya K, Komatsu N, Nakasaki H and Makuuchi H: Recurrence and 5-FU sensitivity of stage II/Dukes' B colorectal cancer with occult neoplastic cells in lymph node sinuses. Oncol Rep 14: 1171-1176, 2005.

13. Mukai M, Sato S, Ninomiya H, Wakui K, Tsuchiya K, Komatsu N, Nakasaki H and Makuuchi H: Recurrence and 5-FU sensitivity of stage II/III node-positive gastric cancer with occult neoplastic cells in lymph node sinuses. Oncol Rep 14: 1505-1510, 2005.

14. Mukai M, Sato S, Ninomiya H, Wakui K, Tsuchiya K, Komatsu N, Nakasaki H and Makuuchi H: Recurrence and 5-FU sensitivity of stage I/II node-negative breast, lung or gastric cancer with occult neoplastic cells in lymph node sinuses. Oncol Rep 15: 815-820, 2006.

15. Mukai M, Sato S, Ninomiya H, Wakui K, Tsuchiya K, Komatsu N, Nakasaki H and Makuuchi H: Prospective study on the recurrence/metastasis of stage II/III colorectal and gastric cancer associated with occult neoplastic cells in lymph node sinuses: three-year interim results. Oncol Rep 16: 405-410, 2006.

16. Mukai M, Oida Y, Tajima T, Kishima K, Ninomiya H, Sato S, Nakamura M, Nakasaki $\mathrm{H}$ and Makuuchi $\mathrm{H}$ : Alternating hepatic arterial infusion and systemic chemotherapy for stage IV colorectal cancer with synchronous liver metastasis. Oncol Rep 16: 865-870, 2006.

17. Mambo NC and Gallager HS: Carcinoma of the breast: the prognostic significance of extranodal extension of axillary disease. Cancer 39: 2280-2285, 1977.

18. Suemasu K and Naruke T: Prognostic significance of extranodal cancer invasion of mediastinal lymph nodes in lung cancer. Jpn J Clin Oncol 12: 207-212, 1982.

19. Watanabe H, Kato H, Tachimori Y, Yamaguchi $\mathrm{H}$ and Itabashi M: Characteristics of the spread pattern for esophageal carcinoma. Kyoubu Geka (Jpn J Thorac Surg) (in Japanese with English abstract) 42: 682-689, 1989.

20. Ueno $\mathrm{H}$ and Mochizuki H: Clinical significance of extra bowel skipped cancer infiltration in rectal cancer. Jpn J Surg 27: 617-622, 1997.

21. Ueno H, Mochizuki H and Tamakuma S: Prognostic significance of extranodal microscopic foci discontinuous with primary lesion in rectal cancer. Dis Colon Rectum 41: 55-61, 1998.

22. Singh AK, Myerson RJ, Birnbaum EH, Fleshman JW, Kodner IJ, Lokett MA and Read TE: Outcome of patients with rectal adenocarcinoma and localized pelvic non-nodal metastatic foci. Dis Colon Rectum 43: 1217-1221, 2000

23. Ueno $\mathrm{H}$, Hase $\mathrm{K}$ and Mochizuki $\mathrm{H}$ : Criteria for extramural perineural invasion as a prognostic factor in rectal cancer. $\mathrm{Br}$ J Surg 88: 994-1000, 2001.

24. Ishikawa K, Hashiguchi Y, Mochizuki H, Ozeki Y and Ueno H: Extranodal cancer deposit at the primary tumor site and the number of pulmonary lesions are useful prognostic factors after surgery for colorectal lung metastases. Dis Colon Rectum 46: 629-636, 2003. 